\title{
Stabilization of Nonlinear Stochastic Discrete Systems
}

\author{
M. Bensoubaya, A. Ferfera and A. Iggidr \\ CONGE Project \\ INRIA Lorraine \& University of Metz (Dept. of Math, CNRS UPRES - A 70-35) \\ I.S.G.M.P. Bât. A, Ile du Saulcy, F-57045 Metz cedex 01, France \\ e.mail :\{bensouba,ferfera,iggidr\}@loria.fr
}

Keywords: Nonlinear discrete systems, stochastic stability, feedback, Lyapunov functions

\section{Introduction}

Let $(\Omega, K, P)$ be a probability field and denote by $X$ the space of $n$-dimensional random vectors. We consider stochastic discrete-time nonlinear control systems of the form

$$
x_{k+1}(\omega)=f\left(x_{k}(\omega), \omega, u\right)
$$

where $x \in X, u \in \mathbb{R}^{m}$, and $f: \mathbb{R}^{n} \times \Omega \times \mathbb{R}^{m} \rightarrow \mathbb{R}^{n}$ is continuous in $x, \mathcal{C}^{2}$ in $u$ and measurable with respect to $\omega$ satisfying $f(0, \omega, 0)=0$.

Our goal is to give a sufficient condition for feedback stabilization of system (1). Stabilization problem of both continuous-time and discrete-time deterministic nonlinear control systems has attracted the interest of an increasing number of authors in the last decades and various techniques have been developed to design stabilizing feedback.

As for stabilization of stochastic nonlinear control systems, only few results are available in the literature. Properties of the solution of the stochastic algebraic Riccati equation intoduced by Wonham ([6]) are used by Gao and Ahmed ([3]) to deal with the stabilizability problem for a class of continuous-time stochastic nonlinear control systems. More recently, by using the Lyapunov functions method, a stochastic version of Artstein-Sontag's theorem is established in [2], while a Jurdjevic-Quinn type theorem is stated in [1].

In this paper we give a sufficient condition for global stabilization of discrete-time stochastic systems of the form (1). This condition is the discrete analogous of the Jurdjevic-Quinn type condition derived in [1] for continuous-time stochastic control systems.

\section{Stability}

Let $L^{r}(\Omega)$ be the set of elements $x$ of $X$ that satisfy $E\left(|x(\omega)|^{r}\right)=\int_{\Omega}|x(\omega)|^{r} P(d \omega)<\infty$ and $L^{\infty}(\Omega)$ be the set of elements of $X$ which are essentially bounded. We set $\|x\|_{r}=\left(\int_{\Omega}\|x(\omega)\|^{r} P(d \omega)\right)^{1 / r}$, for $x \in L^{r}(\Omega)$, and $\|x\|_{\infty}=\inf _{A \in K, P(A)=0} \sup _{\omega \in C_{K}^{A}}\|x(\omega)\|$, for $x \in$ $\left.L^{\infty}(\Omega)\right)$.
Let $h: \mathbb{R}^{n} \times \Omega \longrightarrow \mathbb{R}^{n}$ be a function which is continuous in its first variable $z \in \mathbb{R}^{n}$ and measurable with respect to $\omega \in \Omega$ and consider the system

$$
\left\{\begin{array}{l}
x_{k+1}(\omega)=h\left(x_{k}(\omega), \omega\right) \quad \omega \in \Omega \quad k \in \mathbb{N} \\
h(0, \omega)=0
\end{array}\right.
$$

Definition Let $\mathcal{A}$ be a subset of $X$.

1. The trivial solution of system (2) is stable in probability with respect to $\mathcal{A}$ if for all $\epsilon>0, \alpha>0$, there exists $\delta>0$ such that, if $x_{0} \in \mathcal{A}$ and $P\left\{\omega,\left|x_{0}(\omega)\right|>\right.$ $\delta\}<\delta$ then $P\left\{\omega,\left|x_{k}\left(x_{0}, \omega\right)\right|>\alpha\right\}<\epsilon$ for all $k \geq 0$.

2. It is almost surely attractive (a.s.a) with respect to $\mathcal{A}$ if $\lim _{k \rightarrow \infty} x_{k}\left(x_{0}, \omega\right)=0$ almost everywhere (a.e) for all $x_{0} \in \mathcal{A}$

3. It is attractive in probability with respect to $\mathcal{A}$ if $\lim _{P k \rightarrow \infty} x_{k}\left(x_{0}, \omega\right)=0$ for all $x_{0} \in \mathcal{A}$ (where $\lim _{P}$ denotes the limit in probability).

4. It is asymptotically stable in probability with respect to $\mathcal{A}$ if it is stable and attractive in probability with respect to $\mathcal{A}$.

Remark that the almost surely attractivity (a.s.a) implies the attractivity in probability. Morozan [5] has introduced the method of Lyapunov Function and has proved the following theorem which we shall use to prove our stabilization result.

Theorem 1 ([5]) Let $S(M)=\left\{x \in L^{\infty}(\Omega),\|x\|_{\infty} \leq\right.$ $M\}$. If there exists a continuous function $V: \mathbb{R}^{n} \longrightarrow$ $[0, \infty)$ such that $V(0)=0, V(z)>0$ for all $z \in$ $\mathbb{R}^{n}, z \neq 0$ and $E(V(h(x(\omega), \omega))-V(x(\omega))) \leq 0$ for all $x \in S(M)$, then the trivial solution of system (2) is stable in probabiliy with respect to $S(M)$.

\section{Stabilzation of discrete control systems}

System (1) will be said globally stabilizable if there exists a feedback law $u: \mathbb{R}^{n} \rightarrow \mathbb{R}^{m}$ with $u(0)=0$ such that the zero solution $x_{k} \equiv 0$ of the closed-loop system $x_{k+1}(\omega)=f\left(x_{k}(\omega), \omega, u\left(x_{k}(\omega)\right)\right)$ is globally asymptotically stable in probability.

Let $\tilde{f}: \mathbb{R}^{n} \times \Omega \rightarrow \mathbb{R}^{n}$ be the function defined by

$$
\tilde{f}(x, \omega)=f(x, \omega, 0)
$$

and assume that: 
(h1) The unforced dynamic system $x_{k+1}(\omega)=$ $\tilde{f}\left(x_{k}(\omega), \omega\right)$ is stable in probability and that there exists a $\mathcal{C}^{2}$ function $V(x)>0, x \neq 0, V(0)=0$, such that $\left\{x \in \mathbb{R}^{n} \mid V(x) \leq \xi\right\}$ is compact $\forall \xi>0$ ( $V$ is proper) and $E(V(\tilde{f}(x(\omega), \omega))-V(x(\omega))) \leq 0, \forall x \in X$.

(h2) If $E\left(V\left(\tilde{f}^{k+1}(x(\omega), \omega)\right)-V\left(\tilde{f}^{k}(x(\omega), \omega)\right)\right)=0$ and $E\left(\frac{\partial V}{\partial x}\left(\tilde{f}^{k+1}(x(\omega), \omega)\right) \frac{\partial f}{\partial u}\left(\tilde{f}^{k}(x(\omega), \omega), \omega, 0\right)\right)=0$, $\forall k \in \mathbb{N}$, then $x=0$.

The notation $\tilde{f}^{k}(x(\omega), \omega), k \geq 0$, is defined recursively by $\tilde{f}^{0}(x(\omega), \omega)=x(\omega)$ and $\tilde{f}^{k}(x(\omega), \omega)=$ $\tilde{f}\left(\tilde{f}^{k-1}(x(\omega), \omega), \omega\right)$, for $k \geq 1$.

$V$ being a $\mathcal{C}^{2}$ function, let $\tilde{V}: \mathbb{R}^{n} \times \Omega \times \mathbb{R}^{m} \rightarrow \mathbb{R}$ and $\varphi: \mathbb{R}^{n} \times \mathbb{R}^{m} \times \mathbb{R}^{m} \rightarrow \mathbb{R}$ be defined respectively by

$$
\begin{aligned}
\tilde{V}(x, \omega, u) & =V(f(x, \omega, u)) \\
\varphi(x, \omega, u, v) & =\int_{0}^{1}(1-t) v^{\mathrm{T}} \frac{\partial^{2} \tilde{V}}{\partial u^{2}}(x, \omega, t u) v d t
\end{aligned}
$$

For a fixed $\eta>0$, let $K_{1}(x)$ and $K_{2}(x)$ be any nonnegative continuous real valued functions satisfying $\forall x \in X$

$$
\begin{aligned}
& K_{1}(x) \geq E\left(\sup _{\|u\| \leq \eta,\|v\|=1}|\varphi(x, \omega, u, v)|\right), \\
& K_{2}(x) \geq E\left(\left\|\frac{\partial V}{\partial x}(\tilde{f}(x, \omega)) \frac{\partial f}{\partial u}(x, \omega, 0)\right\|\right),
\end{aligned}
$$

and set

$$
K(x)=\frac{\eta}{\eta K_{1}(x)+K_{2}(x)+1}
$$

Notice that the real valued function $\varphi$ is homegeneous of degree 2 with respect to $v$. Our main result can now be stated as follow.

Theorem 2 Assume that assumptions (h1) and (h2) hold. Then, for any positive constant $\eta$, system (1) is globally asymptotically stabilizable by means of the continuous feedback law

$$
u^{T}(x)=-K(x) E\left(\frac{\partial V}{\partial x}(\tilde{f}(x(\omega), \omega)) \frac{\partial f}{\partial u}(x(\omega), \omega, 0)\right)
$$

which satisfies $\|u(x)\| \leq \eta, \forall x \in X$.

Proof: The inequality $\|u(x)\| \leq \eta$ is an immediate consequence of (7), (8) and (9). Moreover, setting $\Delta V(x(\omega))=V(f(x(\omega), \omega, u(x))-V(x(\omega))$, one gets from (4) and the Taylor expansion formula:

$$
\begin{aligned}
& E(\Delta V(x(\omega)))=E(\tilde{V}(x(\omega), \omega, 0)-V(x(\omega))) \\
& \quad+E\left(\frac{\partial \tilde{V}}{\partial u}(x(\omega), \omega, 0) u(x)\right. \\
& \left.\quad+\int_{0}^{1}(1-t) u^{\mathrm{T}}(x) \frac{\partial^{2} \tilde{V}}{\partial u^{2}}(x(\omega), \omega, t u(x)) u(x) d t\right)
\end{aligned}
$$

Notice that from (3) and (4) one has

$$
\begin{aligned}
\tilde{V}(x(\omega), \omega, 0) & =V(\tilde{f}(x(\omega), \omega)) \\
\frac{\partial \tilde{V}}{\partial u}(x(\omega), \omega, 0) & =\frac{\partial V}{\partial x}(\tilde{f}(x(\omega), \omega)) \frac{\partial f}{\partial u}(x(\omega), \omega, 0)
\end{aligned}
$$

so that, from (5) and (9),

$$
\begin{aligned}
& E(\Delta V(x(\omega)))=E(V(\tilde{f}(x(\omega), \omega))-V(x(\omega))) \\
&-E\left(\frac{\|u(x)\|^{2}}{K(x)}+\varphi(x(\omega), \omega, u(x), u(x))\right)
\end{aligned}
$$

It follows that, for $x \in X$ such that $u(x)=0$ one has $E(\Delta V(x(\omega)))=E(V(\tilde{f}(x(\omega), \omega))-V(x(\omega))) \leq$ 0 and otherwise, from the homogeneity property of $\varphi(x, \omega, u, v)$ with respect to $v$ one gets:

$$
\begin{aligned}
& E(\Delta V(x(\omega)))=E(V(\tilde{f}(x(\omega), \omega))-V(x(\omega))) \\
& -\frac{\|u(x)\|^{2}}{K(x)}\left[1-K(x) E\left(\varphi\left(x, \omega, u(x), \frac{u(x)}{\|u(x)\|}\right)\right)\right]
\end{aligned}
$$

Besides, using (6), (8) and $\|u(x)\| \leq \eta$ one has:

$$
1-K(x) E\left(\varphi\left(x, \omega, u(x), \frac{u(x)}{\|u(x)\|}\right)\right)>0
$$

and so one gets, from the assumption (h1), $E(\Delta V(x(\omega))) \leq 0, \forall x \in X$, which implies, according to Theorem 1, that the feedback law (9) leeds to a stable closed-loop system :

$$
x_{k+1}(\omega)=f\left(x_{k}(\omega), \omega, u\left(x_{k}(\omega)\right)\right)
$$

Now, since $V$ is proper it follows also that all the solutions of (13) are bounded if $x_{0} \in S(M)$. Besides, according to the stochastic version of LaSalle's invariance principle (see [4]) all these solutions converges in probability to the largest invariant set whose support is contained in the locus $\{x \in X \mid E(\Delta V(x(\omega)))=0\}$. Therefore, in order to prove the attractivity of the trivial solution of (13) it must be shown that for any solution $x_{k}$ of (13) with $x_{0} \in S(M)$ and along which $E\left(\Delta V\left(x_{k}(\omega)\right)\right)=0$ for all $k \geq 0$, one has necessarily $x_{k}=0 \forall k \geq 0$. For, one has from(9) and (11) $E(\Delta V(x(\omega)))=0$ iff $E(V(\tilde{f}(x(\omega), \omega))-V(x(\omega)))=0$ and $E\left(\frac{\partial V}{\partial x}(\tilde{f}(x(\omega), \omega)) \frac{\partial f}{\partial u}(x(\omega), \omega, 0)\right)=0$. Hence, from (3) one has $x_{k}(\omega)=\tilde{f}^{k}\left(x_{0}(\omega), \omega\right)$, for all $k \in \mathbb{N}$ and so $E\left(V\left(\tilde{f}^{k+1}\left(x_{0}(\omega), \omega\right)\right)-V\left(\tilde{f}^{k}\left(x_{0}(\omega), \omega\right)\right)\right)=0$ and $E\left(\frac{\partial V}{\partial x}\left(\tilde{f}^{k+1}\left(x_{0}(\omega), \omega\right)\right) \frac{\partial f}{\partial u}\left(\tilde{f}^{k}\left(x_{0}(\omega), \omega\right), \omega, 0\right)\right)=$ 0 , for all $k \in \mathbb{N}$. This, by assumption (h2), implies that $x_{0}=0$ and by the way $x_{k}=0 \forall k \geq 0$ which ends the proof.

\section{References}

[1] M. Bensoubaya, A. Ferfera, and A. Iggidr. A Jurdjevic-Quinn Type Theorem for Stochastic Nonlinear Control Systems, IEEE Trans. Automat. Control (to appear).

[2] R. Chabour et M. Oumoun. On a universal formula for the stabilization of control stochastic nonlinear systems, J. Stoch. Anal. Appl. (to appear) 17 (1999).

[3] Z.Y. Gao and N.U. Ahmed. Feedback stabilizability of nonlinear stochastic systems with statedependent noise, Int. J. Contr. 45 (1987), 729-737.

[4] H.J. Kushner. Introduction to Stochastic Control. New York: Holt, 1971.

[5] T. Morozan. Stability of stochastic discrete systems. J. Math. Analysis App. 23 (1968), 1-9. 
[6] W.M. Wonham. On a matrix Riccati equation of stochastic control, SIAM J. Contr. Optimiz. 6, (1968) 681-697. 